\section{SAT0101 ABATACEPT IN RHEUMATOID ARTHRITIS ASSOCIATED-INTERSTITIAL LUNG DISEASE: SHORT TERM OUTCOME AND PROGNOSTIC FACTORS}

F. Salaffi ${ }^{1}$, M. Tardella ${ }^{1}$, M. Carotti ${ }^{2}$, M. DI Carlo ${ }^{1}$, A. Giovagnoni ${ }^{2}$.

${ }^{1}$ Rheumatological Clinic, Università politecnica delle Marche, Jesi (AN), Italy;

${ }^{2}$ Radiology Department, Università politecnica delle Marche, Ancona, Italy

Background: The prevalence of interstitial lung disease (ILD) in rheumatoid arthritis (RA) varies in the medical literature from $1 \%$ to $67 \%$ and is a major cause of mortality. Previous works have identified increased age, smoking and anticytrullinated protein antibodies (ACPA) titre as risk factors for RA-ILD (RA-ILD). Conventional treatments for RA may lead to a new onset or worsening of RA-ILD, so treatment should be identified to prevent the onset or exacerbation of RA-ILD. Studies have shown that abatacept (ABA) may improve the outcome of RA-ILD. Objectives: The aim of our study is to evaluate ABA effectiveness and safety in patients with RA-ILD.

Methods: We enrolled RA-ILD patients who started treatment with ABA. All patients underwent thoracic high-resolution computed tomography (HRCT) at the beginning of ABA treatment and after 18 months of therapy. HRCT abnormalities were evaluated using a a computer-aided method (CaM). At each visit clinical, laboratory and respiratory function characteristics were collected and the Clinical Disease Activity Index (CDAI) and the Health Assessment Questionnaire Disability Index (HAQ-DI) for disease activity and functional disability were measured. The cohort was divided into three groups based on the CaM-HRCT results: patients with a lung fibrosis progression of $15 \%$ or more were defined as "worsened", those with a reduction of $15 \%$ or more were defined as "improved", all other patients were defined as "stable." The $15 \%$ threshold of change, derived from the CaM assessment, results from the determination of the standard deviation of the mean value change after 18 months of follow-up of the cohort. The multivariate regression model was used to assess the strength of the association between RA characteristics and HRCT response to ABA.

Results: Forty-four patients ( $81 \%$ women) with a mean age of $59.1 \pm 8.0$ years and a mean duration of the disease of $7.5 \pm 3.1$ years were recruited. Twenty-three (52.3\%) patients were positive for ACPA and $28(63.6 \%)$ for rheumatoid factor. Five patients (11.4\%) showed deterioration of ILD, $31(70.6 \%)$ were in the "stable" group, and 7 patients (16.0\%) experienced improvement over a mean follow-up period of 18 months. The factors related to ILD deterioration were use of methotrexate (MTX) $(p=0.0078)$, and current smoking habit $(p=0.0054)$, according to multivariate regression analysis (Table).

Conclusion: Treatment with ABA is associated with an RA-ILD stability or improvement rate of $86.6 \%$ of patients, while the worsening rate is $11.4 \%$. Concomitant treatment with MTX and current smoking habit are factors associated with RA-ILD worsening. MTX discontinuation and smoking cessation should be strongly promoted in patients with RA-ILD.

References:

[1] Salaffi F, Carotti M, Di Carlo M, Tardella M, Giovagnoni A. High-resolution computed tomography of the lung in patients with rheumatoid arthritis: Prevalence of interstitial lung disease involvement and determinants of abnormalities. Medicine (Baltimore). 2019 Sep;98(38):e17088.

[2] Mochizuki T, Ikari K, Yano K, Sato M, Okazaki K. Long-term deterioration of interstitial lung disease in patients with rheumatoid arthritis treated with abatacept. Mod Rheumatol. 2019;29(3):413-417.

Table. Multivariate regression analysis of the variables related to the worsening of RA-ILD

\begin{tabular}{lccccc}
\hline Independent variables & Coefficient & $\begin{array}{c}\text { Std. } \\
\text { Error }\end{array}$ & $\mathbf{t}$ & $\mathbf{P}$ & $\mathbf{r}_{\text {partial }}$ \\
\hline (Constant) & 1.6370 & & & & \\
Age & -0.0012 & 0.0050 & -0.2 & 0.8131 & -0.042 \\
Sex & 0.025 & 0.1 & 0.23 & 0.8161 & 0.042 \\
Disease duration & 0.015 & 0.017 & 0.89 & 0.3799 & 0.15 \\
ACPA positivity & -0.012 & 0.071 & -0.18 & 0.8566 & -0.032 \\
RF positivity & 0.0030 & 0.079 & 0.038 & 0.9694 & 0.0069 \\
Current smokers & -0.34 & 0.11 & -2.99 & 0.0054 & -0.47 \\
MTX use & -0.39 & 0.13 & -2.84 & 0.0078 & -0.45 \\
DLco & 0.0052 & 0.0061 & 0.84 & 0.4030 & 0.15 \\
FVC & -0.0088 & 0.0088 & -1.006 & 0.3224 & -0.17 \\
CAM-HRCT (\% fibrosis) & 0.00082 & 0.0091 & 0.09 & 0.9285 & 0.016 \\
CDAI & -0.0074 & 0.0038 & -1.96 & 0.0588 & -0.33 \\
HAQ-DI & -0.017 & 0.14 & -0.11 & 0.9059 & -0.021 \\
\hline
\end{tabular}

Disclosure of Interests: None declared DOI: 10.1136/annrheumdis-2020-eular.2778

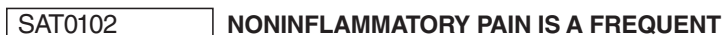 PHENOMENON IN RHEUMATOID ARTHRITIS AND RESPONDS WELL TO TREATMENT WITH SARILUMAB}

E. Choy ${ }^{1}$, V. Bykerk ${ }^{2}$, Y. Lee ${ }^{3}$, G. St John ${ }^{4}$, H. Van Hoogstraten ${ }^{5}$, K. Ford ${ }^{6}$, A. Praestgaard ${ }^{6}$, A. Sebba ${ }^{7} .{ }^{1}$ Cardiff University Hospital, Cardiff, United Kingdom; ${ }^{2}$ Hospital for Special Surgery, New York, NY, United States of America; ${ }^{3}$ Northwestern University, Chicago, IL, United States of America; ${ }^{4}$ Regeneron Pharmaceuticals, Inc., Tarrytown, NY, United States of America; ${ }^{5}$ Sanofi, Bridgewater, NJ, United States of America; ${ }^{6}$ Sanofi Genzyme, Cambridge, MA, United States of America; ${ }^{7}$ University of South Florida, Tampa, FL, United States of America

Background: Inflammation is clearly a key driver of pain in rheumatoid arthritis (RA). However, in some patients the level of pain exceeds what would be expected based on the amount of synovitis observed, which may indicate the presence of noninflammatory pain (NIP). Interleukin-6 (IL-6) has been shown in animal models to increase sensitization to pain and may play a role in NIP. Objectives: To assess the effect of sarilumab, a human IL-6 receptor inhibitor approved for the treatment of adults with moderate to severely active RA, on NIP and disease activity, stratified by baseline (BL) NIP status.

Methods: The analysis included data from three Phase 3 studies of sarilumab: MOBILITY (NCT01061736), MONARCH (NCT02332590), and TARGET (NCT01709578). Patients received double-blind placebo or sarilumab 150 mg or $200 \mathrm{mg}$ subcutaneously (SC) every 2 weeks (q2w), plus weekly csDMARD (MOBILITY and TARGET), or adalimumab $40 \mathrm{mg}$ or sarilumab $200 \mathrm{mg}$ SC q2w as monotherapy (MONARCH).

NIP was defined using an established formula: tender 28-joint count (TJC) - swollen 28-joint count (SJC) $\geq 7$. $^{1,2}$ Patients were assessed for NIP at study BL and fo change in NIP status at Weeks 12 and 24. The proportion of patients achieving ACR20/50/70, Clinical Disease Activity Index (CDAI) $\leq 10$, and DAS28-CRP <3.2 a Week 24 was assessed in patients with and without BL NIP. No inferential statistics were performed.

Results: Of 2112 patients in the analysis, 490 (23\%) met the criteria for NIP at study BL: MOBILITY, $n=294 / 1197$ (25\%); MONARCH, $n=90 / 369$ (24\%); TARGET, $n=106 / 546$ (19\%). BL demographics were similar for patients with or without BL NIP: mean age (SD) was 52.6 (10.7) versus 51.2 (12.3) years, and $85 \%$ versus $81 \%$ were female. Patients with BL NIP had higher CDAI, DAS28 CRP, pain Visual Analog Scale (VAS), and TJC at BL versus patients without NIP (Table). Of patients with NIP at BL, those who received sarilumab were more likely to have no NIP at Weeks 12 and 24 versus patients who received placebo or adalimumab (Figure 1). The percentage of patients achieving improvements in disease activity at Week 24 was greater for sarilumab versus adalimumab among both patients with and without BL NIP, and these differences were larger among patients with BL NIP for all assessments except ACR50 (Figure 2).

Table. Baseline characteristics

\begin{tabular}{lcc}
\hline & \multicolumn{2}{c}{ Patients with TJC - SCJ $\geq 7$} \\
\hline Mean (SD) & Yes $(\mathbf{n}=\mathbf{4 9 0})$ & No $(\mathbf{n}=\mathbf{1 6 2 2})$ \\
\hline Duration of RA, years & $9.1(8.6)$ & $9.7(8.4)$ \\
TJC, 0-28 & $21.7(4.7)$ & $14.3(6.2)$ \\
SJC, 0-28 & $10.7(4.3)$ & $13.1(6.0)$ \\
CRP, mg/L & $22.7(27.0)$ & $22.9(24.0)$ \\
HAQ-DI, 0-3 & $1.8(0.6)$ & $1.7(0.6)$ \\
DAS28-CRP & $6.4(0.7)$ & $5.9(0.9)$ \\
CDAI & $46.0(9.4)$ & $40.4(13.0)$ \\
Pain VAS & $72.3(18.2)$ & $67.0(20.7)$ \\
\hline
\end{tabular}

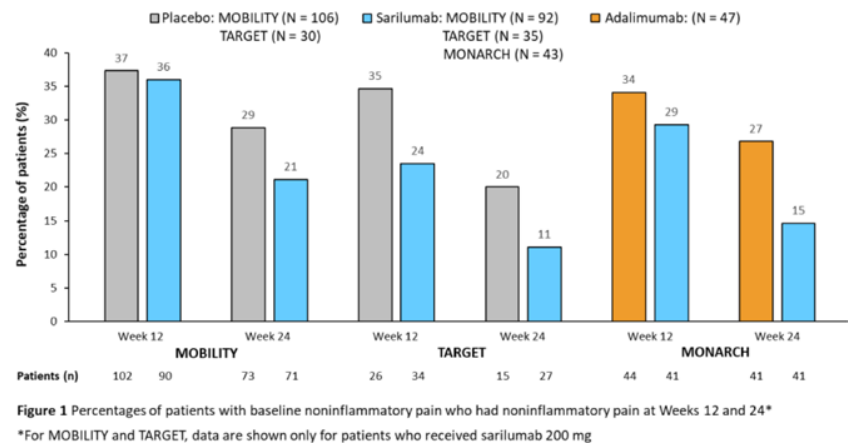

Conclusion: NIP was prevalent at $\mathrm{BL}$ in the patient populations assessed. Among patients with BL NIP, a lower proportion continued to have NIP at Weeks 12 and 